\title{
Hunting for QCD instantons at the LHC in events with large rapidity gaps
}

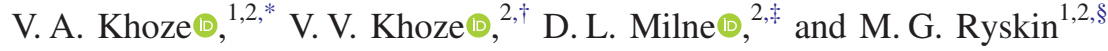 \\ ${ }^{1}$ Petersburg Nuclear Physics Institute, NRC Kurchatov Institute, Gatchina, St. Petersburg 188300, Russia \\ ${ }^{2}$ IPPP, Department of Physics, Durham University, Durham DH1 3LE, United Kingdom
}

(Received 26 April 2021; accepted 19 August 2021; published 14 September 2021)

\begin{abstract}
We outline a strategy of how to search for QCD instantons of invariant mass $20-60 \mathrm{GeV}$ in diffractive events in low-luminosity runs at the LHC. We show that, by imposing appropriate selection criteria on the final states, one can select the kinematic regime where the instanton signal exceeds the background by a factor of at least 8 . In spite of the relatively strong cuts that we impose on the total transverse energy and the number of charged tracks, $\sum_{i} E_{T, i}>15 \mathrm{GeV}$ and $N_{\mathrm{ch}}>20$ measured within the $0<\eta<2$ interval and excluding events with high- $p_{T}$ particles, the expected cross section is sufficiently large to study the instanton production in events with large rapidity gaps at low luminosities, thus avoiding problems with pileup. The paper also includes an updated computation of instanton cross sections and other parameters relevant for ongoing studies.
\end{abstract}

DOI: 10.1103/PhysRevD.104.054013

\section{INTRODUCTION}

Instantons are nonperturbative classical solutions of Euclidean equations of motion in non-Abelian gauge theories [1]. In the semiclassical limit, instantons provide dominant contributions to the path integral and describe quantum tunneling between different vacuum sectors of the theory [2-4]. Over the years, a number of phenomenological models and approaches were developed where QCD instantons either were directly responsible for generating or at least contributed to many key aspects of nonperturbative low-energy dynamics of strong interactions [5-10]. These include the role of instantons in the breaking of the $U(1)_{A}$ symmetry and the spontaneous breakdown of the chiral symmetry, the formation of quark and gluon condensates, topological susceptibility of the vacuum, and the nonperturbative generation of the axion potential. Instanton-based phenomenological models provide at least a qualitative description of the QCD vacuum. ${ }^{1}$ These predictions are in good agreement with lattice calculations [11]; see also [12,13].

\footnotetext{
v.a.khoze@durham.ac.uk

†valya.khoze@durham.ac.uk

*daniel.1.milne@durham.ac.uk

\$ryskin@thd.pnpi.spb.ru
}

${ }^{1}$ Their potential shortcomings originate from attempting to describe the strongly coupled QCD vacuum dynamics using a semiclassical (weak-coupling) approximation where the path integral is expanded around classical instanton background fields.

Published by the American Physical Society under the terms of the Creative Commons Attribution 4.0 International license. Further distribution of this work must maintain attribution to the author(s) and the published article's title, journal citation, and DOI. Funded by SCOAP ${ }^{3}$.
Our motivation here is different. We would like to search for QCD instanton events directly in scattering experiments. This setup provides a more controlled environment than QCD vacuum modeling, as the scattering event kinematics will enable us to remain in a relatively weakly coupled regime where semiclassical instanton methods are reliable. In scattering processes, the instanton can be thought of as new nonperturbative multiparticle vertices in a Feynman diagram, and for its computation at the microscopic level we will use the formalism developed in recent papers [14,15]. QCD instanton-generated processes are predicted to be produced with a large scattering cross section at small center-of-mass partonic energies, although, as shown in Ref. [15], discovering them at hadron colliders using conventional high- $p_{T}$ trigger requirements is a challenging task that requires alternative dedicated search strategies. Developing such a strategy based on the event selection with large rapidity gaps at low luminosities is the purpose of this paper.

In the past, in the context of deep inelastic scattering, QCD instanton contributions [16-18] were searched for (but not observed) $[19,20]$ at the HERA collider.

In QCD, the instanton configuration consists of the gauge field,

$$
A_{\mu}^{a \text { inst }}(x)=\frac{2 \rho^{2}}{g} \frac{\bar{\eta}_{\mu \nu}^{a}\left(x-x_{0}\right)_{\nu}}{\left(x-x_{0}\right)^{2}\left(\left(x-x_{0}\right)^{2}+\rho^{2}\right)},
$$

along with the fermion components for light $\left(m_{f}<1 / \rho\right)$ fermions,

$$
\bar{q}_{L f}=\psi^{(0)}(x), \quad q_{R f}=\psi^{(0)}(x) .
$$

The gauge field $A_{\mu}^{a \text { inst }}$ is the Belavin-Polyakov-SchwartzTyupkin instanton solution [1] of the self-duality equations 
in the singular gauge. Here, $\rho$ is the instanton size and $x_{0}$ is the instanton position. Constant group-theoretic coefficients $\bar{\eta}_{\mu \nu}^{a}$ are the 't Hooft eta symbols defined in Ref. [2]. The fermionic components $\psi^{(0)}$ are the corresponding normalized solutions of the Dirac equation $\gamma^{\mu} D_{\mu}\left[A_{\mu}^{a \text { inst }}\right] \psi^{(0)}=0$. These are the fermion zero modes of the instanton. The instanton configuration is a local minimum of the Euclidean action, and the action on the instanton is given by $S_{I}=\frac{8 \pi^{2}}{g^{2}}=\frac{2 \pi}{\alpha_{s}}$.

The instanton configuration (1) has topological charge equal to one, and, thus, due to the chiral anomaly, the instanton processes violate chirality. If the instanton is produced by a two-gluon initial state, the final state of this instanton-mediated process will have $N_{f}$ pairs of quarks and antiquarks with the same chirality:

$$
g+g \rightarrow n_{g} \times g+\sum_{f=1}^{N_{f}}\left(q_{R f}+\bar{q}_{L f}\right),
$$

where $N_{f}$ is the number of light flavors relative to the inverse instanton size $m_{f}<1 / \rho$. The instanton contribution to the amplitude for this process comes from expanding the corresponding path integral in the instanton field background. At leading order in the instanton perturbation theory, the amplitude takes the form of an integral over the instanton collective coordinates (see, e.g., Refs. [14,15] for more detail):

$$
\begin{aligned}
\mathcal{A}_{2 \rightarrow n_{g}+2 N_{f}}^{\mathrm{LO}}= & \int d^{4} x_{0} \int_{0}^{\infty} d \rho D(\rho) e^{-S_{I}} \prod_{i=1}^{n_{g}+2} A_{\mathrm{LSZ}}^{\mathrm{inst}}\left(p_{i} ; \rho\right) \\
& \times \prod_{j=1}^{2 N_{f}} \psi_{\mathrm{LSZ}}^{(0)}\left(p_{j} ; \rho\right) .
\end{aligned}
$$

The factors of $A_{\mathrm{LSZ}}^{\mathrm{inst}}\left(p_{i} ; \rho\right)$ and $\psi_{\mathrm{LSZ}}^{(0)}\left(p_{j} ; \rho\right)$ are the standard insertions of the Lehmann-Symanzik-Zimmermann (LSZ)reduced instanton fields in the momentum representation, and $D(\rho)$ is given by the known expression for the instanton density [2].

From the point of view of Feynman graphs, the leadingorder instanton amplitude (4) reveals itself as a family of multiparticle vertices (with different numbers of emitted gluons) integrated over the instanton position and size. It describes the emission of a large number of gluons, $n_{g} \propto E^{2} / \alpha_{s}$, together with a fixed number of quarks and antiquarks, one pair for each light flavor in accordance with Eq. (3). The semiclassical suppression factor $\exp \left(-S_{I}\right)=$ $\exp \left(-2 \pi / \alpha_{s}\right)$ will be partially compensated by the growth with jet energy $E$ of the high-multiplicity cross section for the process (3). The fully factorized structure of the field insertions on the right-hand side of Eq. (4) implies that at leading order in instanton perturbation theory there are no correlations between the momenta of the external legs in the instanton amplitude. The momenta of individual particles in the final state are mutually independent, apart from overall momentum conservation.

Thus, to discover the QCD instanton, we have to observe in the final state a multiparticle cluster or a fireball which contains, in general, a large number of isotropically distributed gluon (mini)jets accompanied by $N_{f}$ pairs of light quark jets generated by a subprocess such as Eq. (3).

It is quite challenging, however, to identify the instanton on top of the underlying event. Recall that the instanton is not a particle, and there will be no peak in the invariant mass $M_{\text {inst }}$ distribution. ${ }^{2}$ The mean value of $M_{\text {inst }}$ can at least, in principle, be "measured" or reconstructed as the mass of the minijet system created by the instanton fireball in each given event. Talking about the instanton, we actually mean a family of objects of different sizes $\rho$ and different orientation in the color and the Lorentz spaces. The mean value of $M_{\text {inst }}$ depends on $\rho$, increasing when $\rho$ decreases. Since experimentally it is impossible to measure the instanton size $\rho$, below, we use the mass $M_{\text {inst }}$ to characterize the properties of the instanton production event.

The paper is organized as follows: In Sec. II, we detail the main sources of background and how their cross section behaves with energy. Section III details the physics of events with large rapidity gaps, while Sec. IV explains the rationale behind the selection criteria employed in this paper. Section V updates the calculation of cross sections presented in Ref. [15] to account for small virtualities present in incoming gluons and provides details of the Monte Carlo generation of our background. In Sec. VI, we give our results, and Sec. VII provides a discussion theoretical uncertainties and additional instanton subprocesses. In Sec. VIII, we present our conclusions.

\section{BACKGROUND}

Let us consider the main sources of background which can mimic the instanton signal.

The cross section of instanton production falls steeply with $M_{\text {inst }}$ mainly due to the factor $\exp \left(-S_{I}\right)=$ $\exp \left(-2 \pi / \alpha_{s}(\rho)\right)$ in the amplitude. At smaller values of $\rho$, the instanton action $S_{I}=2 \pi / \alpha_{s}(\rho)$ increases, since the QCD coupling $\alpha_{s}$ decreases. Calculations presented in Sec. V (cf. Table I) show that the elementary cross section of the parton level subprocess (3) falls approximately as

$$
\hat{\sigma}_{\text {inst }} \propto M_{\text {inst }}^{-6},
$$

\footnotetext{
${ }^{2}$ What we mean by the instanton mass is the partonic energy $\sqrt{\hat{s}}$ of the initial two-gluon state in the process (3). As we integrate over the Bjorken $x$ variables when computing the hadronic cross section, we sum over a broad range of instanton masses.
} 
over a broad low-to-intermediate energy range (it becomes less steep, $\hat{\sigma}_{\text {inst }} \propto M_{\text {inst }}^{-4}$, at lower energies $20-30 \mathrm{GeV}$ ).

On the other hand, the dimensionless rate $M^{2} \sigma_{\mathrm{pQCD}}$ of a similar purely perturbative QCD (pQCD) subprocess, $g g \rightarrow N$ minijets, decreases only logarithmically. For the perturbatively formed "hedgehog" configuration of $N$ final state jets, we would expect

$$
\sigma_{\mathrm{pQCD}}(g g \rightarrow N \text { jets }) \sim \frac{16 \pi}{M^{2}}\left(\frac{N_{c}}{\pi} \alpha_{s}(M)\right)^{N}
$$

where $M$ denotes the invariant energy of the perturbatively formed cluster of minijets. Thus, at sufficiently large values of $M_{\text {inst }}$ the instanton signal (5) will become negligible relative to the purely perturbative QCD production (6).

In the regime of interest of moderately small $M_{\text {inst }}$, we face, however, another problem that needs to be addressed. The instanton event can be mimicked by the multiple parton interactions (MPIs) illustrated in Fig. 1. Indeed, the double (triple, $\ldots, n$ ) parton scattering produces a few pairs of jets (dijets) which would look like a fireball, thus obscuring the final state signature of the genuine instanton signal.

The cross section of such a process can be evaluated as

$$
\frac{d \sigma}{d E_{1}^{2} \ldots d E_{n}^{2}} \sim\left(\frac{d \sigma}{\sigma_{\mathrm{eff}} d E_{1}^{2}} \cdots \frac{d \sigma}{\sigma_{\mathrm{eff}} d E_{n}^{2}}\right) \sigma_{\mathrm{eff}}
$$

where $E_{i}$ denotes the transverse energy of a jet in the $i$ dijet system, $\sigma_{\text {eff }} \sim 10 \mathrm{mb}$ (see Fig. 4 in Ref. [21] and references therein) and the cross section $d \sigma / d E_{i}^{2} \sim \pi \alpha_{s}^{2} / E_{i}^{4}$. That is, at low $M_{\text {inst }}$ when the transverse energies of produced jets become small, the MPI processes will dominate. It was shown in Ref. [22] that the events caused by such multiple parton interactions manifest high sphericity $S$, similar to the instanton signal. Our strategy to suppress them is outlined in the following section and involves a final state selection with large rapidity gaps.

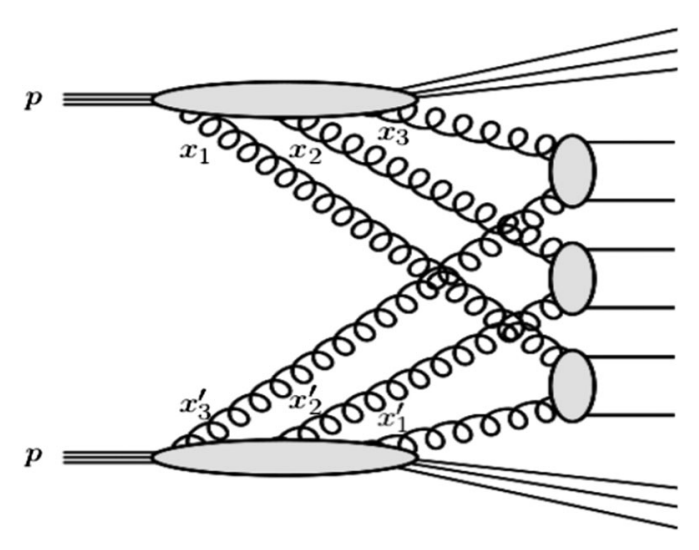

FIG. 1. Schematic diagram for the triple parton scattering process.
Finally, we would like to emphasize one additional point. Since our knowledge on the dynamics of confinement and hadronization is quite limited, general purpose Monte Carlo event generators introduce certain phenomenological parameters that are tuned in order to better describe the experimental data (for a review, see, e.g., Ref. [23]). But if a low-mass instanton contributes to the scattering, its presence will affect the data, and after such tuning we lose the possibility to distinguish the low- $M_{\text {inst }}$ instanton from other hadronization effects.

\section{EVENTS WITH LARGE RAPIDITY GAPS}

As discussed above, we expect a large "underlying event" background in the region of both large and small instanton masses. However, the low- $M_{\text {inst }}$ background caused by multiparton interactions can be effectively suppressed by selecting events with large rapidity gaps (LRGs). Indeed, each additional "parton-parton $\rightarrow$ dijet" scattering is accompanied by the color flow created by the parton cascade needed to form the incoming partons (see Fig. 1). This color flow produces secondaries which fill the LRGs. The LRG survival probability $S^{2}$ (i.e., the probability not to destroy the LRG) is rather small: $S^{2} \leq 0.1$; see, e.g., [24]. Thus, the probability to observe $n$ additional branches of parton-parton interactions in LRG events is suppressed by the factor $\left(S^{2}\right)^{n}$.

Moreover, recall that the events with an LRG mainly occur at large values of $b_{t}$ (the separation between the two incoming protons in the transverse plane), where the proton optical density (opacity) is small $[24,25]$. On the other hand, the MPI (double/triple/.../n parton scattering) processes proceed dominantly at small values of $b_{t}$, where the proton opacity is much larger. Thus, we expect that the suppression factor $S^{2}$ for each additional scattering should be smaller than the average value of $S^{2}$ discussed in Ref. [24]. Therefore, in this paper, when evaluating backgrounds for relatively lowmass instantons in the events with an LRG, to a first approximation we neglect the MPI contributions.

\section{SEARCH STRATEGY}

We propose searching for the instanton as a multiparticle cluster or fireball with a mass of about $20-60 \mathrm{GeV}$ in the events with an LRG. The presence of an LRG can be detected either by detecting the leading forward proton with beam momentum fraction, $x_{L}=1-\xi$, very close to 1 $\left(\xi=x_{\text {Pom }} \leq 0.01\right)$, or by observing no hadron activity in the forward calorimeters.

Since the relatively heavy instanton produces a rather large number of jets, we are looking for high-multiplicity events which

(i) do not contain high- $E_{T}$ jets and

(ii) still have a large density of the transverse energy, $\sum_{i} d E_{T i} / d \eta \sim M_{\text {inst }} / 3$ (the sum is over all secondary particles in the given $\eta$ interval). 
TABLE I. The instanton production cross section for the subprocess (3) for a range of partonic center-of-mass system energies $E$ (i.e., instanton masses) and the mean number of emitted gluons in addition to the mean value of $1 / \rho$ and $\alpha_{s}(1 / \rho)$. For energies larger than $150 \mathrm{GeV}$, the suppression coming from the $3 \mathrm{GeV}$ virtuality is fairly negligible, and one can use the numbers given in Ref. [15]. Note that the mean value of $R / \rho$ was $\sim 1.55$ at all energies.

\begin{tabular}{lllll}
\hline \hline$E[\mathrm{GeV}]$ & $\hat{\sigma}(g g \rightarrow I)[\mathrm{pb}]$ & $\langle 1 / \rho\rangle[\mathrm{GeV}]$ & $\alpha_{s}(1 / \rho)$ & $\left\langle n_{g}\right\rangle$ \\
\hline 20 & $2.01 \times 10^{6}$ & 1.69 & 0.327 & 7.81 \\
25 & $9.49 \times 10^{5}$ & 1.98 & 0.306 & 8.58 \\
30 & $4.64 \times 10^{5}$ & 2.27 & 0.290 & 9.07 \\
35 & $2.32 \times 10^{5}$ & 2.52 & 0.279 & 9.61 \\
40 & $1.25 \times 10^{5}$ & 2.84 & 0.267 & 9.67 \\
50 & $3.89 \times 10^{4}$ & 3.38 & 0.251 & 10.56 \\
60 & $1.38 \times 10^{4}$ & 3.87 & 0.241 & 10.89 \\
70 & $5.45 \times 10^{3}$ & 4.33 & 0.232 & 11.38 \\
80 & $2.36 \times 10^{3}$ & 4.85 & 0.224 & 11.67 \\
90 & $1.08 \times 10^{3}$ & 5.24 & 0.219 & 12.31 \\
100 & $5.44 \times 10^{2}$ & 5.82 & 0.213 & 12.10 \\
110 & $2.79 \times 10^{2}$ & 6.21 & 0.209 & 12.62 \\
120 & $1.53 \times 10^{2}$ & 6.71 & 0.205 & 12.77 \\
130 & $8.56 \times 10^{1}$ & 7.13 & 0.201 & 13.04 \\
140 & $4.99 \times 10^{1}$ & 7.57 & 0.198 & 13.25 \\
150 & $3.01 \times 10^{1}$ & 8.00 & 0.195 & 13.45 \\
\hline \hline
\end{tabular}

Indeed, from Table I, we see that the instanton of mass $30 \mathrm{GeV}$ produces about 17 jets (nine gluons plus four light $\bar{q} q$ pairs). The energy of each jet $E_{T i} \sim 1 / \rho \sim 2 \mathrm{GeV}$. After hadronization, in such an event we expect about 40-60 particles. The large multiplicity can be used as the main (or additional) trigger to select the events of interest.

Note that the elementary (parton level) instanton cross section is predicted to be rather large $(\sim 1 \mu \mathrm{b})$, while the background due to hedgehoglike pQCD multijet production (6) is much smaller-already for the case of six final jets, the expected elementary (hedgehog QCD) cross section $\sim \mathrm{few} \cdot \mathrm{nb}$ which can be safely neglected.

On the other hand, in the events with an LRG, the probability to observe such large multiplicity in a limited rapidity interval falls steeply with $N_{\text {track }}$ (see, e.g., Fig. 13 in Ref. [25]). Moreover, since each jet from the instanton cluster contains a leading hadron with $p_{T} \geq 1 \mathrm{GeV}$, we can select the events with a large multiplicity [say, $N_{c h}\left(p_{T}>0.5 \mathrm{GeV}\right)>20$ ] of high- $p_{T}$ particles, in this way strongly suppressing the soft QCD background.

Since the LHC detectors never cover the whole $(4 \pi)$ rapidity interval, there is no chance to adequately measure the value of $M_{\text {inst }}$. To select the events with appropriate $M_{\text {inst }}$ we introduce the cut on the total transverse energy measured within the given rapidity interval $\sum_{i} E_{T i}>M_{0}$.
Here, we consider the instanton production in the Pomeron-proton collision (in terms of Regge theory, the Pomeron exchange is responsible for the presence of the LRG) selecting events with a large multiplicity and relatively large transverse energy. ${ }^{3}$ We expect that these events will be more or less spherically symmetric; that is, in such events there should be a large probability to observe the sphericity $S$ close to 1 . Since we cannot observe the particles in the whole $4 \pi$ sphere, we consider the "transverse sphericity" (or cylindricity) defined as $S_{T}=2 \lambda_{2} /\left(\lambda_{1}+\lambda_{2}\right)$, where $\lambda_{i}$ are the eigenvalues of the matrix

$$
S^{\alpha \beta}=\frac{\sum_{i} p_{i}^{\alpha} p_{i}^{\beta}}{\sum_{i}\left|\vec{p}_{i}^{2}\right|}
$$

and $\lambda_{2}<\lambda_{1}$. Here, $p_{i}^{\alpha}$ is the two-dimensional transverse component of the momentum of the $i$ th particle, and we sum over all particles observed in the event within a given rapidity interval.

Finally, recall that the decay of the instanton produces one additional pair of each flavor of light $\left(m_{f}<1 / \rho\right)$ quark. So, in the case of the signal, we expect to observe a larger number of strange and charm particles than in background events.

\section{CALCULATION}

\section{A. Parton-level instanton cross sections}

The instanton cross sections of the elementary gluoninitiated process (3) are calculated following the approach developed in recent papers $[14,15]$. In this approach, the total parton-level instanton cross section for the process $g g \rightarrow X$ is related by the optical theorem to the imaginary part of the forward elastic scattering amplitude computed in the background of the instanton-anti-instanton $(I \bar{I})$ configuration:

$$
\hat{\sigma}_{\text {inst }}=\frac{1}{E^{2}} \operatorname{Im} \mathcal{A}_{4}^{I \bar{I}}\left(p_{1}, p_{2},-p_{1},-p_{2}\right) .
$$

Here, $E=\sqrt{\left(p_{1}+p_{2}\right)^{2}}$ denotes the invariant mass $\sqrt{\hat{s}}$ of the two-gluon initial state. The resulting instanton cross section takes the form of the finite-dimensional integral [15] over the instanton and anti-instanton collective coordinates (scale sizes $\rho$ and $\bar{\rho}$, the separation between the instanton and anti-instanton centers, $R$, and the relative $I \bar{I}$ orientations $\Omega$ ):

\footnotetext{
${ }^{3}$ The idea to search for the instanton in events with very large multiplicity but not too large transverse energy and the first evaluation of the instanton cross section at collider energies was discussed long ago in Ref. [26].
} 


$$
\begin{aligned}
\hat{\sigma}_{\mathrm{tot}}^{\text {inst }} \simeq & \frac{1}{E^{2}} \operatorname{Im} \frac{\kappa^{2} \pi^{4}}{36 \cdot 4} \int \frac{d \rho}{\rho^{5}} \int \frac{d \bar{\rho}}{\bar{\rho}^{5}} \int d^{4} R \int d \Omega\left(\frac{2 \pi}{\alpha_{s}\left(\mu_{r}\right)}\right)^{14}\left(\rho^{2} E\right)^{2}\left(\bar{\rho}^{2} E\right)^{2} \mathcal{K}_{\text {ferm }}(z) \\
& \times\left(\rho \bar{\rho} \mu_{r}^{2}\right)^{b_{0}} \exp \left(R_{0} E-\frac{4 \pi}{\alpha_{s}\left(\mu_{r}\right)} \mathcal{S}(z)-\frac{\alpha_{s}\left(\mu_{r}\right)}{16 \pi}\left(\rho^{2}+\bar{\rho}^{2}\right) E^{2} \log \frac{E^{2}}{\mu_{r}^{2}}-Q(\rho+\bar{\rho})\right) .
\end{aligned}
$$

In the above integral, $\kappa^{2}$ denotes the known normalization constant, $\mathcal{K}_{\text {ferm }}(z)$ is the factor arising from the overlap of fermion zero modes of $I$ and $\bar{I}$, and the variable $z$ is a certain conformally invariant ratio of the collective coordinates $R, \rho$, and $\bar{\rho}$ $[27,28]$ :

$$
z=\frac{R^{2}+\rho^{2}+\bar{\rho}^{2}+\sqrt{\left(R^{2}+\rho^{2}+\bar{\rho}^{2}\right)^{2}-4 \rho^{2} \bar{\rho}^{2}}}{2 \rho \bar{\rho}} .
$$

The first factor in the exponent in Eq. (10) describes the energy input from the initial state, the second factor is the action of the instanton-anti-instanton configuration $[28] S_{I \bar{I}}(z)=\frac{4 \pi}{\alpha_{s}} \mathcal{S}(z)$, where

$$
\mathcal{S}(z)=3 \frac{6 z^{2}-14}{(z-1 / z)^{2}}-17-3 \log (z)\left(\frac{(z-5 / z)(z+1 / z)^{2}}{(z-1 / z)^{3}}-1\right) \text {. }
$$

The third term in the exponent,

$$
\exp \left(-\frac{\alpha_{s}\left(\mu_{r}\right)}{16 \pi}\left(\rho^{2}+\bar{\rho}^{2}\right) E^{2} \log \frac{E^{2}}{\mu_{r}^{2}}\right),
$$

is the quantum effect that takes into account resummed perturbative exchanges between the hard initial-state gluons and the instantons [29]. Inclusion of these quantum effects is required in order to resolve the well-known infrared problem in the $\rho, \bar{\rho} \rightarrow \infty$ limit, as QCD instantons with $\rho \gg\left(\frac{16 \pi}{\alpha_{s}} \frac{1}{E^{2} \log E^{2}}\right)^{1 / 2}$ are automatically cut off by these quantum corrections.

We note that the initial-state partons (in our case gluons) are not strictly on mass shell but carry small order-GeV virtualities $Q_{1}$ and $Q_{2}$. For the gluon emitted from the Pomeron, we choose $Q_{1}=2 \mathrm{GeV}$, and the second gluon that originated from the proton has the virtuality $Q_{2}=1 \mathrm{GeV}$.

From the Pomeron side, the expected virtuality $Q_{1}^{2} \simeq q_{t}^{2}$ is close to the mean transverse momentum squared, $q_{t}^{2}$, of the gluon inside the Pomeron. Its value can be estimated as $q_{t}^{2} \sim 1 / \alpha_{P}^{\prime} \sim 4 \mathrm{GeV}^{2}$, where we take the Pomeron trajectory slope $\alpha_{p}^{\prime}=0.25 \mathrm{GeV}^{-2}$ [30]. The gluon virtuality in the proton parton distribution function (PDF), $Q_{2} \sim 1 \mathrm{GeV}$, was chosen for the following reasons. As a rule, the global parton analysis based on Dokshitzer-Gribov-Lipatov-Altarelli-Parisi evolution starts the evolution at some $Q=Q_{0} \geq 1 \mathrm{GeV}$. We do not know the parton distributions at a smaller $Q$ (it is not even clear whether we can use the parton language at such small $Q$ ). Note, however, that the low- $x$ gluon density increases as the scale $Q$ increases. So we consider the value of $Q_{2}=1 \mathrm{GeV}$ as a conservative estimate. These virtualities introduce the form factor $^{4} e^{-Q \rho}$ in the instanton vertex, with $Q=$ $Q_{1}+Q_{2}=3 \mathrm{GeV}$ in our case. This is the origin of the final term

$$
\exp (-Q(\rho+\bar{\rho}))
$$

in the exponent of the cross section in Eq. (10).

To further simplify the integrand, we select a natural value for the renormalization scale dictated by the inverse instanton size. This prescription removes the large $\left(\rho \mu_{r}\right)^{b_{0}}\left(\bar{\rho} \mu_{r}\right)^{b_{0}}$ factor in front of the exponent in Eq. (10). Hence, we choose

$$
\mu_{r}=1 /\langle\rho\rangle=1 / \sqrt{\rho \bar{\rho}} .
$$

For the reference point of $\alpha_{s}$, we choose its value at the $\tau$ mass, $\alpha_{s}\left(m_{\tau}\right)=0.32$ following Refs. [31,32] that fits the experimental data $[33,34]$. The choice of $m_{\tau}$ as the reference scale for $\alpha_{s}$ is conveniently close to the interesting (for us) regime of relatively light instantons with sizes $1 /\langle\rho\rangle \sim$ few GeV. For the running coupling at energy scales above $m_{\tau}$, we use the one-loop expression, while for the lower scales we freeze the coupling at a fixed critical value $\alpha_{s}=0.35$ :

$\frac{4 \pi}{\alpha_{s}(\langle\rho\rangle)} \simeq \begin{cases}\frac{4 \pi}{0.32}-2 b_{0} \log \left(\langle\rho\rangle m_{\tau}\right) & \text { for }\langle\rho\rangle^{-1} \geq 1.45 \mathrm{GeV} \\ \frac{4 \pi}{0.35} & \text { for }\langle\rho\rangle^{-1}<1.45 \mathrm{GeV} .\end{cases}$

\footnotetext{
${ }^{4}$ The form factor is a direct consequence of Fourier transforming the instanton field to momentum space to obtain $A_{\text {LSZ }}^{\text {inst }}\left(p_{i}\right)$, where the momentum $p_{i}$ has the virtuality $Q_{i}^{2}$ [28] and was explored and used extensively in the context of deep inelastic scattering in Refs. [16,18].
} 
At these energy scales, we are in the regime of

$$
N_{f}=4,
$$

active quarks, and this is the $N_{f}$ value we use in $b_{0}=$ $11-2 N_{f} / 3$ and in the instanton density expressions for $\kappa^{2}$ and $\mathcal{K}_{\text {ferm }}$ in Eq. (10).

The rationale for freezing the coupling at 0.35 is that the perturbative evolution of strong coupling at the order of $\sim 1 \mathrm{GeV}$ scale is known to be in conflict with observations. The strong coupling values extracted from the fits to charmonium spectrum give $\alpha_{s} \simeq 0.35$ at the $1-1.2 \mathrm{GeV}$ scale [35], which is close to the measured value $\alpha_{s}\left(m_{\tau}\right)$ at the considerably higher scale $m_{\tau}=$ $1.777 \mathrm{GeV}$ and is about $50 \%$ below the prediction of the two-loop perturbative running. These considerations justify freezing the strong coupling in the infrared at a critical value $\alpha_{s}=0.35$, the approach we will follow in accordance with Eq. (16).

The authors of Ref. [15] evaluated the integral in Eq. (10) numerically using the PYTHON package SciPy [36] to derive the parton-level instanton cross sections along with the mean number of gluons in the final state as functions of partonic energy $E$. In this work, we follow the computational approach of Ref. [15], except that we now also account for the effect of $Q \simeq 3 \mathrm{GeV}$ gluon virtualities (14) from the outset and use the prescription (16) for the running coupling.

The data in Table I show that the instanton production cross section $\hat{\sigma}_{\text {inst }}$ is sharply suppressed at high partonic energies (high instanton masses) and becomes large at low values of $E$. This is also consistent with the earlier calculations in Ref. [14] that used the same theory setup for the parton-level instanton cross section (10) but evaluated the integral in the steepest descent approximation. The sharp suppression of the instanton cross section at large partonic energies is the consequence of the inclusion of the quantum exchanges between the hard initial gluons that resulted in the exponential form factor (13). It cuts off the (anti-)instanton sizes more and more efficiently when $E$ increases, ultimately leading to the integral being dominated by smaller and smaller instantons, thus exponentially suppressing the overall cross section, in agreement with the results shown in Table 1 and Fig. 6 in Ref. [14]. In the low-energy limit, contributions of large instantons with $\rho \gg 1 /(3 \mathrm{GeV})$ are cut off by gluon virtualities in Eq. (14).

For more detail on the theory formalism leading to Eq. (10) and the resulting evaluation of $\hat{\sigma}_{\text {inst }}(E)$ and $\left\langle n_{g}\right\rangle(E)$, we refer the reader to Ref. [15].

\section{B. Hadronic cross sections for signal and background}

The cross section of instanton production in protonproton collisions reads

$$
\begin{aligned}
\sigma_{p p \rightarrow I}= & \int_{\hat{s}_{\text {min }}}^{s_{p p}} d x_{1} d x_{2} f\left(x_{1}, Q_{1}^{2}\right) f\left(x_{2}, Q_{2}^{2}\right) \\
& \times \hat{\sigma}_{\text {inst }}\left(E^{2}=x_{1} x_{2} s_{p p}\right),
\end{aligned}
$$

where $s_{p p}$ is the center-of-mass energy of the hadron collider and $\hat{\sigma}$ is the partonic instanton cross section. Selecting the events with an LRG or the leading proton, we have to replace one PDF, say, $f\left(x_{1}, Q_{1}^{2}\right)$, which describes the probability to find an appropriate parton (gluon) in the incoming proton, by the so-called diffractive PDF, $f^{D}\left(x_{1}, Q_{1}^{2}\right)$, which describes the corresponding distribution in the Pomeron.

The lower limit $\hat{s}_{\min }$ in the integral (18) is introduced for technical reasons. Strictly speaking, one is supposed to integrate from $\hat{s}_{\min }=0$ to $s_{p p}$. However, instantons of masses much smaller than $20 \mathrm{GeV}$ are not under theoretical control, and the semiclassical dilute instanton approximation becomes invalid for large instantons with $\rho \sim \mathrm{GeV}$, where the theory can no longer be considered weakly coupled. ${ }^{5}$ It is impossible to fix $\hat{s}_{\min }$ experimentally; instead, we have to choose and impose experimental cuts on final states that will suppress the contribution of the low-mass instantons. In practice, we will set $\sqrt{\hat{s}_{\min }}=20 \mathrm{GeV}$ in the integral ${ }^{6}$ and then impose the cuts.

Instanton samples are generated using the RAMBO algorithm of [37] and then showered and hadronized using PYTHIA8 [38]. The scale of the process was taken to be $1 /\langle\rho\rangle$ for the purposes of showering in PYTHIA. As already mentioned, in the integral (18) we set the minimum instanton mass generated to be $\hat{s}_{\min }=20 \mathrm{GeV}$. However, we also note that the inclusion of these low-mass instantons is expected to give a negligible contribution with our choice of selection criteria.

Background events were simulated by generating inelastic pion-proton collisions (see Fig. 2) in PYTHIA for the proton energy $E_{p}=7 \mathrm{TeV}$ and the Pomeron energy $E_{\text {Pom }}=7 \cdot x_{\text {Pom }} \mathrm{TeV}$ (with a pion being used to simulate a Pomeron) and $x_{\text {Pom }}$ fixed at 0.003 . For the proton PDF, we use the NNPDF3.1luxQED NNLO [39-42] set, while for the parton distribution in the Pomeron (mimicked in PYTHIA by the pion) the H1 fit B [43] is used. The $Q^{2}$ evolution for the Pomeron PDF is performed by QCDNUM [44].

When computing the signal, the instanton cross section calculated in Eq. (18) was multiplied by the gap survival

\footnotetext{
${ }^{5}$ Furthermore, when $\rho \gtrsim 0.3 \mathrm{fm} \sim 1.5 \mathrm{GeV}^{-1}$, one can no longer distinguish between the instantons created in collisions and the instantonlike configurations populating the QCD ground state [11]. The role of $\hat{s}_{\text {min }}$ is to cut off such large ground-state field configurations.

${ }^{6}$ Taking $\hat{s}_{\min }=(20 \mathrm{GeV})^{2}$ gives a hadronic instanton cross section of $1.72 \mu \mathrm{b}$ (after multiplying by $S^{2}$ and taking the factorization scale as $1 /\langle\rho\rangle)$.
} 


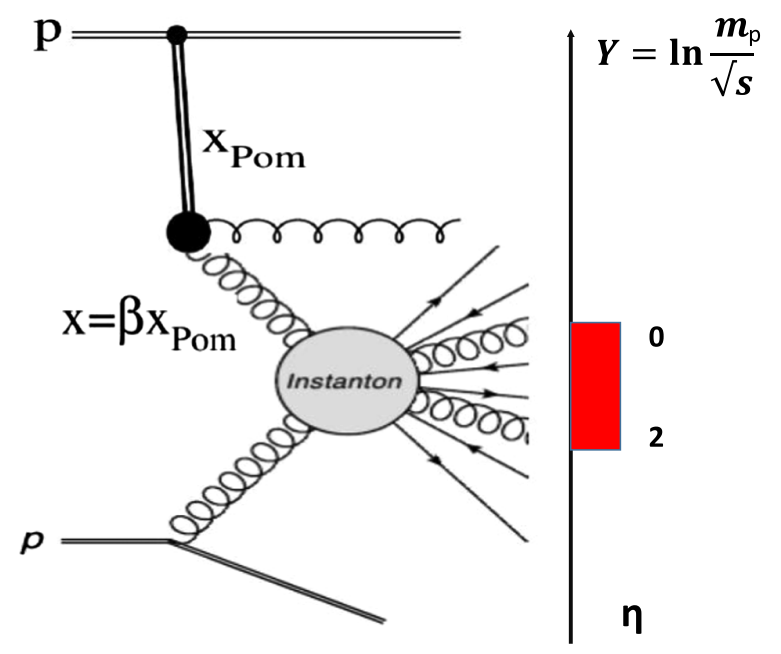

FIG. 2. Instanton production in a diffractive process with an LRG. The Pomeron exchange is shown by the thick doubled line. The red bar shows the range of $\eta$ considered in this paper. $Y$ indicates the incoming proton position in rapidity. As shown in the diagram, secondaries will be produced outside this range but will not be used when calculating $E_{T}$ or $N_{c h}$.

probability $S^{2}$. For simplicity, we took $S^{2}=0.1$ in agreement with the experimental results $[45,46]$ for diffractive high- $E_{T}$ dijet production. The background cross section was normalized to the experimental yield of single dissociation $d \sigma^{S D} / d \ln \left(1 / x_{\text {Pom }}\right) \simeq 0.15 \mathrm{mb}$ [47]. In generating both the instanton and the background samples, multiparton interactions were disabled in PYTHIA.

Since in the case of a soft QCD background, only a very small fraction of events satisfy the $\sum_{i} E_{T i}>M_{0} \mathrm{GeV}_{\mathrm{cut}}{ }^{7}$ (which we had used further), we generate the "hard QCD" events with a minimum transverse momentum of $10 \mathrm{GeV}$ using PYTHIA. Using lower- $p_{T}$ samples from PYTHIA, we concluded that the contribution of lower- $p_{T}$ events is negligible.

\section{RESULTS}

As an example, we consider instanton production at $14 \mathrm{TeV}$ in the events with the leading proton momentum fraction $x_{L}=1-x_{\text {Pom }}=0.997$. That is, we calculate the cross section $d \sigma / d \ln \left(1 / x_{\text {Pom }}\right)$ at $x_{\text {Pom }}=0.003$. This may

\footnotetext{
${ }^{7}$ Actually, to get a large $\sum_{i} E_{T i}$ in a perturbative QCD event, we have to produce a high- $E_{T}$ jet or to consider the event with a large number of multiple parton interactions. The last possibility is strongly suppressed by the LRG survival factor.
}

be considered as the total observed cross section when and if one integrates over the interval of $x_{\text {Pom }}$ from 0.0018 to 0.005 [i.e., $\Delta \ln \left(x_{\text {pom }}\right)$ interval equal to 1$]^{8}$

Our results are presented in Figs. 3 and 4. To further suppress the background, we impose the following set of experimental selection criteria that look realistic for the present ATLAS and CMS detectors. We exclude the very low- $p_{T}$ particles and consider only the secondary hadrons with transverse momentum $p_{T}>0.5 \mathrm{GeV}$ produced within the $0<\eta<2$ rapidity interval, as shown in Fig. 2. We select the events for which the total transverse energy of the charged secondaries (with $p_{T}>0.5 \mathrm{GeV}$ and $0<\eta<2)^{9}$ is $\sum_{i} E_{T, i}>15 \mathrm{GeV}$ and the number of corresponding charged tracks $N_{c h}>20$. We also demand that there are no charged particles in this region with $p_{T}>$ $2 \mathrm{GeV}$ (Figs. 3 and 4, left). Plots on the right-hand side in Figs. 3 and 4 implement the constraint that no charged particles are present in the region $-2<\eta<2$ with $p_{T}>2.5 \mathrm{GeV}$.

As is seen in Fig. 4, after the proposed cuts the instanton signal exceeds the background by a factor of over (left) 8 and (right) 4 and for $S_{T}>0.5$ the signal to background ratio $\mathrm{S} / \mathrm{B}$ is even higher. Notably, in the $N_{c h}>20$ region the expected cross section is still rather large $(\sim 1 \mathrm{nb})$. This allows one to observe the instanton production in lowluminosity runs without pileup problems.

The sphericity distribution is shown in Fig. 4. It is clearly seen that while the background sphericity falls for $S_{T}>0.5$ the instanton signal is mainly concentrated at $S_{T} \sim 0.7-0.8$. For $S_{T}>0.8$, the expected instanton contribution is a factor of 10 greater than the background (for both sets of selection criteria).

Finally, Fig. 5 shows the distribution over the number of neutral kaons. ${ }^{10}$ The selection criteria used are the same as those described in Fig. 3. As expected, the kaon multiplicity is larger in the events containing the instanton, and for $N_{K^{0}}>5$ the S/B ratio is about 5 (for both sets of selection criteria).

\footnotetext{
${ }^{8}$ Note that in this region the dependence of single dissociation cross section $d \sigma / d \ln \left(1 / x_{\mathrm{Pom}}\right)$ is practically flat as is shown in Refs. [25,47,48].

${ }^{9}$ We shifted the $\eta$ interval in the direction opposite to the LRG (or the leading proton which has the negative rapidity) in order not to have gluons with too large $x$ (where the gluon density rapidly decreases with increasing $x$ ) from the Pomeron PDF. Recall that for $x_{\text {Pom }}=0.003$ the energy of the "incoming" Pomeron is $21 \mathrm{GeV}$ only.

${ }^{10}$ This can be observed via the $\left(K^{0}\right)_{S} \rightarrow \pi^{+} \pi^{-}$decay.
} 

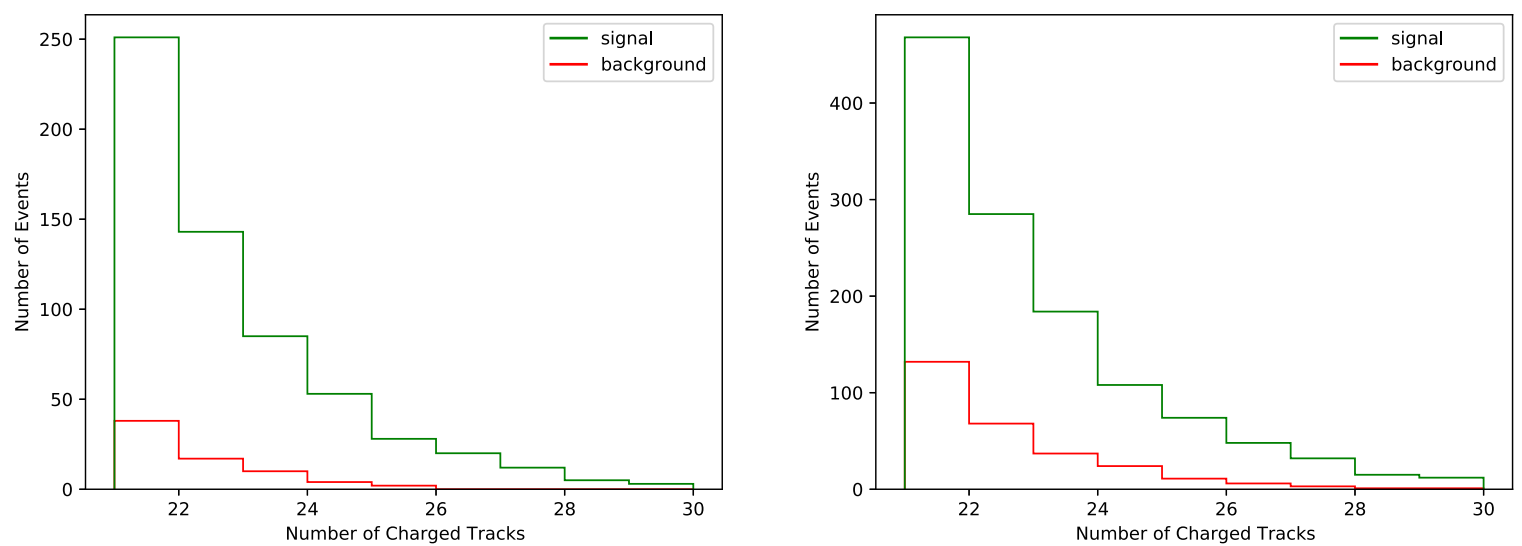

FIG. 3. Multiplicity distribution of charged hadrons produced in the events with the instanton (green line) in comparison with the expected background (red line). The number of events is normalized to the integrated luminosity $L=1 \mathrm{pb}^{-1}$ and $\Delta \ln \left(x_{\mathrm{Pom}}\right)=1$ interval. We required events to have $\sum_{i} E_{T, i}>15 \mathrm{GeV}$ and $N_{c h}>20$, summing only over charged particles in the region $0<\eta<2$ with $p_{T}>0.5 \mathrm{GeV}$, with an additional constraint that there is no charged particle in this region with $p_{T}>2 \mathrm{GeV}$ (left figure) or no charged particle in the region $-2<\eta<2$ with $p_{T}>2.5 \mathrm{GeV}$ (right figure).
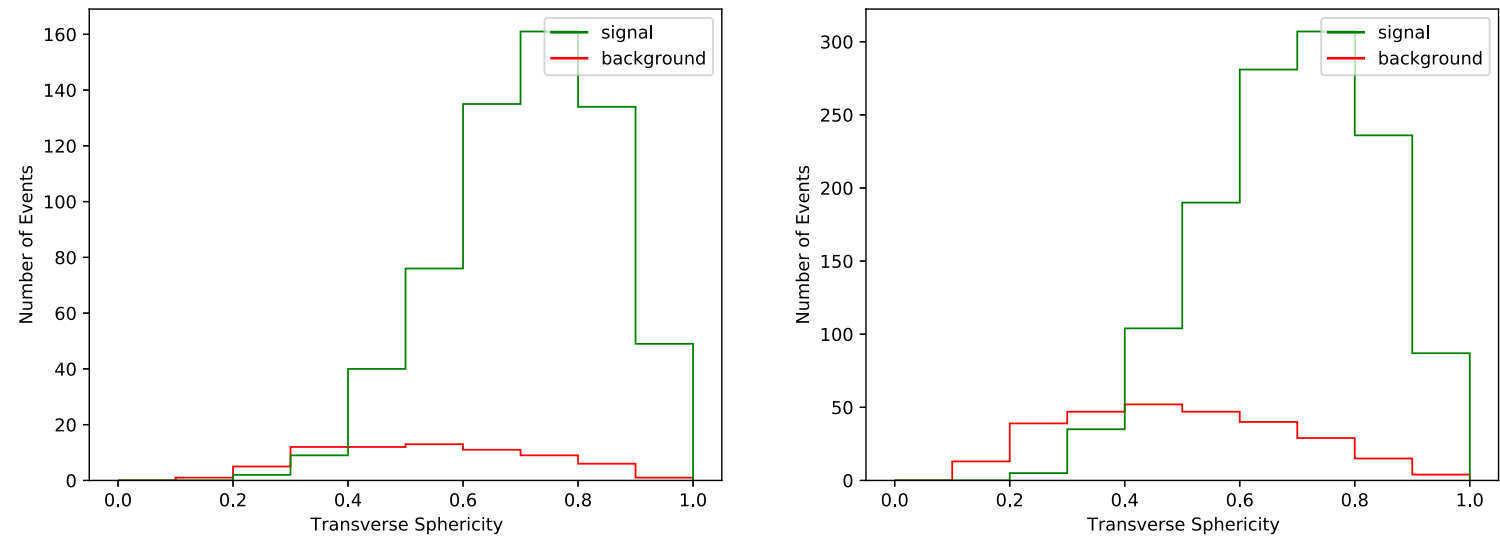

FIG. 4. Distribution over the transverse sphericity $S_{T}$ [Eq. (8)] of the charged hadrons produced in the events with the instanton (green line) in comparison with the expected background (red line). The selection criteria used are the same as those described in Fig. 3.
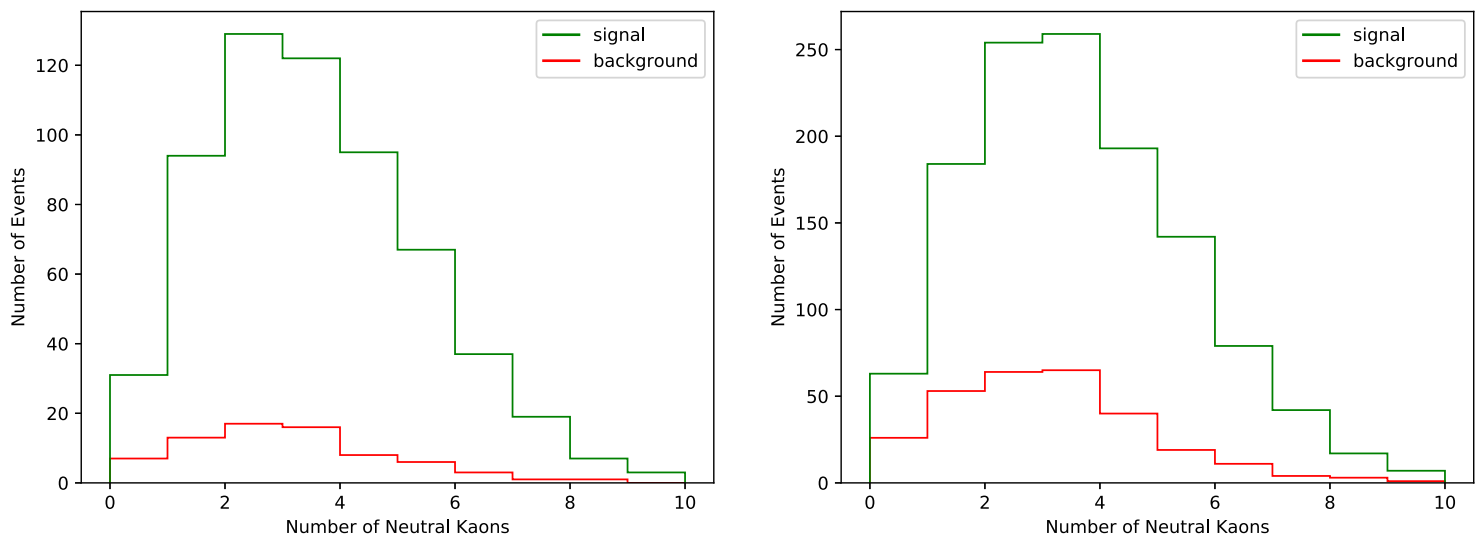

FIG. 5. Distribution over the number of neutral kaons produced in the events with the instanton (green line) in comparison with the expected background (red line). The selection criteria used are the same as those described in Fig. 3. 


\section{DISCUSSION}

The possibility of direct experimental observation of QCD instantons at hadron colliders has recently attracted a fair amount of attention [14,15,49]. The authors of Ref. [15] evaluated the sensitivity of the LHC and Tevatron high- and low-luminosity runs, and Ref. [49] obtained a first limit based on existing minimum bias data.

Extraction of the instanton signal on top of the underlying event at the LHC, not relying on events with large rapidity gaps, is a challenging task. One problem, not considered in Ref. [49], is how to select in the experiment the events corresponding to the chosen $M_{\text {inst }}$ interval. It is impossible to distinguish the jet emitted by the instanton from the jet created during the parton showering or due to the presence of a perturbative QCD multijet hedgehog contribution (the latter is another problem that has not been addressed until now).

The idea to observe instantons in the Pomeron-induced process, namely, in the central exclusive production,

$$
p p \rightarrow p+X+p,
$$

was first put forward in Ref. [50] (see also [51]). This was prompted by the reported UA8 observation [52] of the large enhancement in the Pomeron-Pomeron cross section at invariant masses $2<M<5 \mathrm{GeV}$ with roughly isotropic distribution of secondaries. Unfortunately, we (present authors) do not have theoretical control over such low-mass instantons or, for that matter, other nonperturbative contributions in the infrared. In these settings, there is no external mass parameter, and it is not clear how to distinguish instanton production from the background or, for example, from glueball production. On the other hand, searching for a larger-mass instanton in Pomeron-Pomeron collisions has no advantage in comparison with instanton production in events with one LRG, but in the case of Pomeron-Pomeron collisions the expected cross section is much smaller.

\section{A. Other instanton production subprocesses}

Up to now, we were concentrating on only the twogluon-initiated process (3), but one should also consider other quark-, antiquark-, and gluon-initiated two-parton processes, such as

$$
\begin{array}{r}
g+u_{L} \rightarrow n_{g} \times g+u_{R}+\sum_{f=1}^{N_{f}-1}\left(q_{R f}+\bar{q}_{L f}\right), \\
u_{L}+\bar{u}_{R} \rightarrow n_{g} \times g+\sum_{f=1}^{N_{f}-1}\left(q_{R f}+\bar{q}_{L f}\right), \\
u_{L}+d_{L} \rightarrow n_{g} \times g+u_{R}+d_{R}+\sum_{f=1}^{N_{f}-2}\left(q_{R f}+\bar{q}_{L f}\right),
\end{array}
$$

and also include the contributions from multiparton initial states.

We first consider the two-parton-initiated processes. Note that there is no interference between all these subprocesses, since the fermion content of the external states in Eqs. (3) and (20)-(22) is different. Thus, the total contribution comes from adding the individual cross sections from these processes, in other words, summing over the integrals (18) with the appropriate choice of PDFs for each of the partons in the initial state. Since each of these contributions is manifestly positive, our already computed contribution from the gluon fusion process (3) cannot be reduced. It is also clear that one cannot expect any sufficient enhancement to it from accounting for the additional channels (20)-(22). This is because for the parton-level cross section most of the expression in Eq. (10), in particular, its exponent, remains the same; ${ }^{11}$ only the four field insertions for the external states vary. On the other hand, as we have seen, the dominant contributions to hadronic cross sections come from the low- $x$ region, and, as a result, our gluon fusion instanton subprocess is expected to be the dominant one.

Next, we would like to discuss the effects of multiparton initial states in the instanton production. To do this, we would like to distinguish between the two cases: one, where multipartons entering the instanton have originated from just the two parent partons, and the second case, where the multiparton initial state has a genuine multiparton origin, with all three or four partons being emitted from the proton and/or the Pomeron.

The first case, where the multipartons in the initial state can be traced to just two parent partons, is already accounted for by the resummed quantum corrections from the initial states. Such effects are precisely what is included into the initial-initial-state and the initial-final-state propagators in the instanton background computed in Ref. [29]. Our approach takes into account the initial-initial (i.e., hard-hard) quantum effects by explicitly including the Mueller form-factor term (13). The initial-final-state (or hard-soft) interactions were not directly included in our approach; ${ }^{12}$ however, the form factor arising from all such quantum corrections is of the same generic exponential form [29]:

$$
\exp \left(-\frac{\alpha_{s}\left(\mu_{r}\right)}{16 \pi} \rho^{2} E^{2} \sum_{i, j} a_{i j} \log a_{i j}\right), \text { where } a_{i j}:=p_{i} \cdot p_{j} / E^{2},
$$

\footnotetext{
${ }^{11} \mathrm{Up}$ to now, we have included only the initial-state corrections arising from the high-energy limit of the gluon propagator in the instanton background, but, as was shown in Ref. [53], the same result (13) is expected to hold also for fermions in the initial state.

${ }^{12}$ This is done to avoid double counting, since the radiative exchanges involving final-state partons are already accounted for by the optical theorem.
} 
as the initial-initial-state form factor in Eq. (13). Hence, we do not expect a significant difference in the final result from the inclusion of these effects.

The second case corresponds to genuine multiparton initial states, and it is accounted for by adding the contribution to the total cross section from the integrals of the type (18), now with multiple PDFs, one for each parton in the initial state. Like in the two-particle case, discussed earlier, this is a manifestly positive contribution that cannot destructively interfere with our two-gluon contribution. In summary, we expect that a more careful inclusion of all instanton production subprocesses could only enhance the gluon fusion contribution (and only moderately).

\section{B. Theory uncertainties}

Note that all the present calculations should not be considered as the precise theoretical predictions. There are a number of uncertainties.

First of all, one could expect sizable corrections to the assumed factorization of instanton and anti-instanton collective coordinate integration measure in the forward elastic scattering amplitude (10), which strictly holds only at infinitely large interinstanton separations $R^{2} /(\rho \bar{\rho}) \gg 1$. At finite separations, the integration measure was shown in Ref. [27] to satisfy

$$
d \mu_{\bar{I}}=d \mu_{I} d \mu_{\bar{I}}\left(1+\mathcal{O}\left(\frac{1}{z^{2}}\right)\right),
$$

where $z$ is given by Eq. (11). In our calculation, the mean value of $R / \rho$ was $\simeq 1.55$ at all energies, which corresponds to $z \simeq 4.16$, or $1 / z^{2} \simeq 0.058$, which is a reassuringly small value to be optimistic about the validity of the approximate factorization of instanton integration measures (and, thus, the factorization of the determinants of quadratic fluctuation operators in the instanton-anti-instanton background).

Hence, in terms of the conformal $z^{2}$ variable, the individual instanton and the anti-instanton are reasonably well separated. For this reason, we are similarly confident about the validity of the instanton-anti-instanton action expression (12) that was computed for a particular exactly solvable model [27,28] of the instanton-anti-instanton valley configuration. What is more difficult to estimate, however, is the role played by the unknown higher-order perturbative corrections to the resummed quantum exchanges from the initial states in Eq. (13).

Furthermore, there is a rather large theoretical uncertainty in evaluation of the gap survival factor $S^{2}$, which also depends on the details of the particular subprocess (see [24]). Therefore, we prefer to use the experimental value measured in diffractive dijet production $[45,46]$. We evaluate the corresponding uncertainty as $\pm 50 \%$.
We should also note a potentially strong dependence of the predicted cross section on the values of the renormalization $\mu_{R}$ and factorization $\mu_{F}$ scales. In particular, it was found in Ref. [54] that replacing the natural scale $\mu_{F}=1 / \rho$ by the value of $\mu_{F}=M_{\text {inst }}$ enhances the instanton production cross section for instantons of mass larger than $M_{\text {inst }}=$ $50 \mathrm{GeV}$ computed in Ref. [14] by approximately a factor of 5 .

Besides this, as we have already noted, the present calculations are based on the simplest $g g \rightarrow$ instanton subprocess neglecting the possibility to produce the instanton in collision with quarks or with a pair of gluons, like $g+g g \rightarrow$ instanton and so on. Based on our earlier discussion in Sec. VII.1, we consider it unlikely that these new subprocesses would change the qualitative features of the expected signal, but they may enlarge the final cross section.

\section{CONCLUSIONS}

We propose to search for QCD instantons at the LHC in events with large rapidity gaps, and, in this paper, we carried out the first preliminary investigation of this search strategy. These LRG events are selected either by detecting the forward leading proton with $x_{L}$ very close to 1 or by observing no hadron activity in the forward calorimeter. We discussed the main sources of background for the low- and the high-mass instantons and showed that background for the low-mass instantons, which comes mainly from multiple parton interactions, is effectively suppressed in the LRG events by the small gap survival factor $S^{2} \leq 0.1$. This allowed us to find the kinematical domain where the signal from $M_{\text {inst }} \sim 20-60 \mathrm{GeV}$ instantons reliably exceeds the background.

Even with these rather strong cuts in place, the expected instanton cross section remains sufficiently large $(\sim 1 \mathrm{nb})$ to effectively produce and probe QCD instantons at the LHC, at low-luminosity runs, avoiding pileup problems.

\section{ACKNOWLEDGMENTS}

We thank Valery Schegelsky and Michael Spannowsky for useful discussions. V. V. K. is supported by the STFC under Grant No. ST/P001246/1. D. L. M. is supported by an STFC studentship.

Note added.-One may wonder if our instanton results might be compared to the discussion of baryon-numberviolating processes. The authors of Ref. [55] considered a different theory (electroweak rather than QCD in our case), where the general behavior of instanton cross sections with energy is known to be different and should not be compared directly. In fact, as two of the present authors have shown in Ref. [53], when our methods are applied to the electroweak sector, we do get a qualitative agreement with the results of Ref. [55]. As far as QCD instantons are 
concerned, it should also be noted that the dependence of the cross section on the instanton mass at low energies required the knowledge of the prefactor as well as of the leading-order quantum effects (Mueller corrections) that we computed, while the discussion in Ref. [55] concerns only the function in the exponent.
[1] A. A. Belavin, A. M. Polyakov, A. S. Schwartz, and Y. S. Tyupkin, Phys. Lett. B 59, 85 (1975).

[2] G. 't Hooft, Phys. Rev. D 14, 3432 (1976); 18, 2199(E) (1978).

[3] C. G. Callan, Jr., R. F. Dashen, and D. J. Gross, Phys. Lett. B 63, 334 (1976).

[4] R. Jackiw and C. Rebbi, Phys. Rev. Lett. 37, 172 (1976).

[5] G. 't Hooft, Phys. Rep. 142, 357 (1986).

[6] C. G. Callan, Jr., R. F. Dashen, and D. J. Gross, Phys. Rev. D 17, 2717 (1978).

[7] V. A. Novikov, M. A. Shifman, A. I. Vainshtein, and V. I. Zakharov, Nucl. Phys. B191, 301 (1981).

[8] E. V. Shuryak, Nucl. Phys. B203, 116 (1982).

[9] D. Diakonov and V. Y. Petrov, Phys. Lett. B 147, 351 (1984); Nucl. Phys. B272, 457 (1986).

[10] T. Schäfer and E. V. Shuryak, Rev. Mod. Phys. 70, 323 (1998).

[11] A. Hasenfratz and C. Nieter, Phys. Lett. B 439, 366 (1998).

[12] T. A. DeGrand, A. Hasenfratz, and T. G. Kovacs, Nucl. Phys. B505, 417 (1997).

[13] D. A. Smith et al. (UKQCD Collaboration), Phys. Rev. D 58, 014505 (1998).

[14] V. V. Khoze, F. Krauss, and M. Schott, J. High Energy Phys. 04 (2020) 201.

[15] V. V. Khoze, D. L. Milne, and M. Spannowsky, Phys. Rev. D 103, 014017 (2021).

[16] I. I. Balitsky and V. M. Braun, Phys. Lett. B 314, 237 (1993).

[17] S. Moch, A. Ringwald, and F. Schrempp, Nucl. Phys. B507, 134 (1997).

[18] A. Ringwald and F. Schrempp, Phys. Lett. B 438, 217 (1998).

[19] C. Adloff et al. (H1 Collaboration), Eur. Phys. J. C 25, 495 (2002).

[20] S. Chekanov et al. (ZEUS Collaboration), Eur. Phys. J. C 34, 255 (2004).

[21] M. Aaboud et al. (ATLAS Collaboration), Phys. Lett. B 790, 595 (2019).

[22] M. Sas and J. Schoppink, Nucl. Phys. A1011, 122195 (2021).

[23] A. Buckley, J. Butterworth, S. Gieseke, D. Grellscheid, S. Hoche, H. Hoeth, F. Krauss, L. Lonnblad, E. Nurse, P. Richardson et al., Phys. Rep. 504, 145 (2011).

[24] V. A. Khoze, A. D. Martin, and M. G. Ryskin, J. Phys. G 45, 053002 (2018).

[25] V. A. Khoze, A. D. Martin, and M. G. Ryskin, Eur. Phys. J. C 81, 175 (2021).

[26] I. I. Balitsky and M. G. Ryskin, Yad. Fiz. 56N8, 196 (1993) [Phys. At. Nucl. 56, 1106 (1993)]; Phys. Lett. B 296, 185 (1992).
[27] A. V. Yung, Nucl. Phys. B297, 47 (1988).

[28] V. V. Khoze and A. Ringwald, Phys. Lett. B 259, 106 (1991); Valley trajectories in gauge theories, Report No. CERN-TH-6082-91, 1991, https://lib-extopc.kek.jp/ preprints/PDF/1991/9106/9106100.pdf.

[29] A. H. Mueller, Nucl. Phys. B348, 310 (1991); B353, 44 (1991).

[30] A. Donnachie and P. V. Landshoff, Nucl. Phys. B231, 189 (1984).

[31] M. Beneke and M. Jamin, J. High Energy Phys. 09 (2008) 044.

[32] G. Abbas, B. Ananthanarayan, I. Caprini, and J. Fischer, Phys. Rev. D 87, 014008 (2013).

[33] S. Schael et al. (ALEPH Collaboration), Phys. Rep. 421, 191 (2005).

[34] K. Ackerstaff et al. (OPAL Collaboration), Eur. Phys. J. C 7, 571 (1999).

[35] A. M. Badalian and V.L. Morgunov, Phys. Rev. D 60, 116008 (1999).

[36] P. Virtanen, R. Gommers, T. E. Oliphant, M. Haberland, T. Reddy, D. Cournapeau, E. Burovski, P. Peterson, W. Weckesser, J. Bright et al., Nat. Methods 17, 261 (2020).

[37] R. Kleiss, W. J. Stirling, and S. D. Ellis, Comput. Phys. Commun. 40, 359 (1986).

[38] T. Sjöstrand, S. Ask, J. R. Christiansen, R. Corke, N. Desai, P. Ilten, S. Mrenna, S. Prestel, C. O. Rasmussen, and P.Z. Skands, Comput. Phys. Commun. 191, 159 (2015).

[39] A. Buckley, J. Ferrando, S. Lloyd, K. Nordström, B. Page, M. Rüfenacht, M. Schönherr, and G. Watt, Eur. Phys. J. C 75, 132 (2015).

[40] V. Bertone et al. (NNPDF Collaboration), SciPost Phys. 5, 008 (2018).

[41] A. V. Manohar, P. Nason, G. P. Salam, and G. Zanderighi, J. High Energy Phys. 12 (2017) 046.

[42] A. Manohar, P. Nason, G. P. Salam, and G. Zanderighi, Phys. Rev. Lett. 117, 242002 (2016).

[43] A. Aktas et al. (H1 Collaboration), Eur. Phys. J. C 48, 715 (2006).

[44] M. Botje, Comput. Phys. Commun. 182, 490 (2011).

[45] A. M. Sirunyan et al. (CMS and TOTEM Collaborations), Eur. Phys. J. C 80, 1164 (2020).

[46] G. Aad et al. (ATLAS Collaboration), Phys. Lett. B 754, 214 (2016).

[47] G. Aad et al. (ATLAS Collaboration), J. High Energy Phys. 02 (2020) 042; 10 (2020) 182(E).

[48] V. Khachatryan et al. (CMS Collaboration), Phys. Rev. D 92, 012003 (2015).

[49] S. Amoroso, D. Kar, and M. Schott, Eur. Phys. J. C 81, 624 (2021). 
[50] E. Shuryak and I. Zahed, Phys. Rev. D 68, 034001 (2003).

[51] E. Shuryak and I. Zahed, arXiv:2102.00256.

[52] A. Brandt et al. (UA8 Collaboration), Eur. Phys. J. C 25, 361 (2002).
[53] V. V. Khoze and D. L. Milne, Int. J. Mod. Phys. A 36, 2150032 (2021).

[54] M. Mangano, arXiv:2101.02719.

[55] F. L. Bezrukov, D. Levkov, C. Rebbi, V. A. Rubakov, and P. Tinyakov, Phys. Lett. B 574, 75 (2003). 